\title{
Effects of an Exercise Training Program in Physical Condition After Liver Transplantation in Familial Amyloidotic Polyneuropathy: A Case Report
}

\author{
M.T. Tomás, H. Santa-Clara, E. Monteiro, E. Barroso, and L. Bettencourt Sardinha
}

\begin{abstract}
From the Faculty of Human Kinetics-Technical University of Lisbon (M.T.T., H.S.-C., L.B.S.), Exercise and Health Laboratory; Higher School of Health Technology (M.T.T.), Lisbon, Polytechnique Institute; and Hepatobiliopancreatic and Transplantation Center (E.M., E.B.), Curry Cabral Hospital, Lisbon, Portugal.
\end{abstract}

Address reprint requests to Teresa Tomás, Faculty of Human Kinetics-Technical University of Lisbon, Estrada da Costa, Cruz Quebrada, Lisbon,Portugal.E-mail:mttomas@fmh.utl.pt or teresa.tomas@estesl.ipl.pt

\section{ABSTRACT}

Introduction. Familial amyloidotic polyneuropathy (FAP) is a neurodegenerative disease that leads to sensory and motor polyneuropathies as well as functional limitations. So far, liver transplantation is the only treatment for FAP because the mutated protein causing the disease is mainly produced in the liver. With the increasing survival of transplant recipients, functional and cardiovascular problems as consequences of immunosuppressant side effects are increasing associated with sedentary lifestyles and/or pretransplantation status. We sought to analyze the impact of exercise training programs on 1 FAP patient's course long-term after liver transplantation.

Methodology. A FAP patient (female; 49 years of age; body mass index _ $18.8 \mathrm{~kg} / \mathrm{m}_{2}$ ) underwent a liver transplantation 133 months before assessment. She was assessed for body composition, isometric quadriceps muscle strength, functional capacity, fatigue, and levels of physical activity before and after a 6-month period of combined exercise training.

Results. After the exercise training program, almost all variables were improved, namely, total body skeletal muscle mass, proximal femoral bone mineral density, quadriceps strength, maximal oxygen consumption on 6 minutes walk test $(6 \mathrm{mwt})$ or $\mathrm{VO}_{2 \text { peak, }}$ total ventilation on $6 \mathrm{mwt}$, and fatigue. The improvement in distance on $6 \mathrm{mwt}(69.2 \mathrm{~m})$ was clinically significant. Preintervention the levels of physical activity were below international recommendations for health; after the program they achieved the recommendations.

Conclusion. The results showed an improvement in functional capacity with a decrease in future disability risk associated with a better lifestyle with respect to physical activity levels in 1 patient.

(C) 2011 by Elsevier Inc. All rights reserved.

360 Park Avenue South, New York, NY 10010-1710

Transplantation Proceedings, 43, 257-258 (2011) 